\title{
Surgical repair of sciatic nerve traumatic rupture: technical considerations and approaches
}

\author{
Hussam Abou-AI-Shaar, MD, Nam Yoon, MD, and Mark A. Mahan, MD \\ Department of Neurosurgery, Clinical Neurosciences Center, University of Utah, Salt Lake City, Utah
}

Traumatic proximal sciatic nerve rupture poses surgical repair dilemmas. Disruption often causes a large nerve gap after proximal neuroma and distal scar removal. Also, autologous graft material to bridge the segmental defect may be insufficient, given the sciatic nerve diameter. The authors utilized knee flexion to allow single neurorrhaphy repair of a large sciatic nerve defect, bringing healthy proximal stump to healthy distal segment. To avoid aberrant regeneration, the authors split the sciatic nerve into common peroneal and tibial divisions. After 3 months, the patient can fully extend the knee and has evidence of distal regeneration and nerve continuity without substantial injury.

The video can be found here: https://youtu.be/lsezRT5I8MU.

KEYWORDS sciatic nerve; nerve rupture; knee mobilization; neuroma; neurorrhaphy; video 